TRANSACTIONS OF THE

AMERICAN MATHEMATICAL SOCIETY

Volume 354, Number 8, Pages 3263-3273

S 0002-9947(02)02900-8

Article electronically published on February 20, 2002

\title{
ON THE CENTERED HARDY-LITTLEWOOD MAXIMAL OPERATOR
}

\author{
ANTONIOS D. MELAS
}

\begin{abstract}
We will study the centered Hardy-Littlewood maximal operator acting on positive linear combinations of Dirac deltas. We will use this to obtain improvements in both the lower and upper bounds or the best constant $C$ in the $L^{1} \rightarrow$ weak $L^{1}$ inequality for this operator. In fact we will show that $\frac{11+\sqrt{61}}{12}=1.5675208 \ldots \leq C \leq \frac{5}{3}=1.66 \ldots$.
\end{abstract}

\section{INTRODUCTION}

The centered Hardy-Littlewood maximal operator acting on $L^{1}(\mathbb{R})$, is defined by

$$
M f(x)=\sup _{h>0} \frac{1}{2 h} \int_{x-h}^{x+h}|f| .
$$

As is well known this nonlinear operator maps $L^{1}(\mathbb{R})$ into the Lorenz space $L^{1, \infty}(\mathbb{R})$ of weak $L^{1}$ functions and is bounded. It is therefore natural to ask for the best constant $C$ such that for every $f \in L^{1}(\mathbb{R})$ and every $\lambda>0$,

$$
|\{M f>\lambda\}| \leq \frac{C}{\lambda}\|f\|_{1} .
$$

The exact value of $C$ is not known. A. Carbery proposed that $C=3 / 2([2$, Problem 7.74c) a joint conjecture with F. Soria which also appears in [9] and corresponds to sums of equidistributed Dirac deltas. This conjecture has been refuted by J. M. Aldaz in [1] who obtained the lower bounds $C \geq \frac{23}{15}=1.533 \ldots$ (and also $\geq \frac{37}{24}=$ $1.541 \ldots)$. Using an iteration scheme that is based on Aldaz' construction and leads to a certain dynamical system J. Manfredi and F. Soria obtained the lower bound (8], see also [1]):

$$
C \geq \frac{5}{3}-\frac{2 \sqrt{7}}{3} \sin \left(\frac{\arctan (3 \sqrt{3})^{-1}}{3}\right)=1.5549581 \ldots
$$

If one considers the uncentered maximal operator, then the best constant is actually equal to 2 and this holds not only for the Lebesgue measure but also for any positive measure in $\mathbb{R}$ (see [3]). In this case the best constants for the corresponding $L^{p} \rightarrow L^{p}$ theorems (for $p>1$ ) are also known (see [6]).

However to even prove that the constant for the centered maximal operator is strictly less than the corresponding constant for the uncentered maximal operator, that is $C<2$, which was asked in [9], requires more delicate analysis. This was done by J. M. Aldaz in [1] where he proved that $C \leq \frac{9+\sqrt{41}}{8}=1.9253905 \ldots$.

Received by the editors March 14, 2000 and, in revised form, June 15, 2001.

2000 Mathematics Subject Classification. Primary 42B25. 
The purpose of this paper is to obtain a deeper understanding of the maximal operator. This will allow us to get better bounds for $C$, both lower and upper. More preciesely we will prove the following.

Theorem 1. For the best constant $C$ in the weak $(1,1)$ inequality of the centered Hardy-Littlewood maximal operator we have

$$
\frac{11+\sqrt{61}}{12} \leq C \leq \frac{5}{3} .
$$

Hence this theorem gives $1.5675208 \ldots \leq C \leq 1.66 \ldots$ which improves the previously known bounds. However we feel that the lower bound given in Theorem 1 is the exact value of $C$.

To prove this theorem we will use the discretization technique introduced by $\mathrm{M}$. de Guzmán [7] and used in [4, [9], [10] and 1]. The main point of this technique applied to the present situation is the fact that M. de Guzmán result as sharpened by M. Trinidad Menárguez and F. Soria (see Theorem 1 in [9]) implies that the best constant $C$ in inequality (1.2) is equal to the corresponding best constant in the inequality

$$
|\{M \mu>\lambda\}| \leq \frac{C}{\lambda} \int_{\mathbb{R}} d \mu
$$

where $\lambda>0$ and $\mu$ runs through all measures of the form $\sum_{i=1}^{n} \delta_{t_{i}}$ for $n \geq 1$ and $t_{1}, \ldots, t_{n} \in \mathbb{R}$, where for any positive measure $\mu$

$$
M \mu(x)=\sup _{h>0} \frac{1}{2 h} \int_{x-h}^{x+h} d \mu .
$$

To prove Theorem 1 we will first, in Section 2, transform the problem to one of combinatrial-analytic nature that has to do with a certain covering of the set $\{M \mu \geq 1 / 2\}$ which can be assumed connected. In Section 3 we introduce what we will call the gap interval by collapsing certain subintervals of $\{M \mu \geq 1 / 2\}$. This new interval will have a covering whose overlapping properties will reflect the covering properties of our initial cover of $\{M \mu \geq 1 / 2\}$. In Section 4 we will give a construction that leads to our lower bound. Then in Section 5 we will take advantage of the overlapping properties of certain coverings of the gap interval to obtain our upper bound.

\section{Definitions and Preliminaries}

In view of the discretization result of M. de Guzmán and M. Trinidad Menárguez and F. Soria described in the introduction it will be enough to study measures of the form

$$
\mu=\sum_{i=1}^{n} k_{i} \delta_{y_{i}}
$$

(slightly more general than sums of Dirac deltas) where $n$ is a positive integer, $k_{1}, \ldots, k_{n}$ are nonnegative real numbers and the real numbers $\left\{y_{i}\right\}$, positions, satisfy $y_{1} \leq \ldots \leq y_{n}$. Moreover if $k_{1}, \ldots, k_{n}>0$ and $y_{1}<\ldots<y_{n}$ we would say that $\mu$ is written in reduced form. Obviously every measure as in (2.1) has a unique reduced form which is the expression (2.1) for $\mu$ with the smallest possible $n$. 
By scaling the $k_{i}$ 's it is enough to study inequality (1.4) only for $\lambda=1 / 2$. Hence for any measure as in (2.1) we define the set

$$
E(\mu)=\left\{x: M \mu(x) \geq \frac{1}{2}\right\}
$$

and the norm of $\mu$,

$$
N(\mu)=\int_{\mathbb{R}} d \mu=k_{1}+\ldots+k_{n}
$$

Then in view of inequality (1.4) we also define the ratio of $\mu$ by

$$
R(\mu)=\frac{|E(\mu)|}{2 N(\mu)} .
$$

Then the constant $C$ in Theorem 1 is the supremum of all numbers $R(\mu)$ as $\mu$ runs through all positive measures of the form (2.1).

Now we will give a description of the set $E(\mu)$ which will be used in all that will follow.

Lemma 1. For any measure $\mu$ of the form 2.1 and every $x \in \mathbb{R}$ we have

$$
M \mu(x)=\max \left\{\frac{k_{i}+\ldots+k_{j}}{\left|2 x-y_{i}-y_{j}\right|+y_{j}-y_{i}}: 1 \leq i \leq j \leq n\right\}
$$

(with the convention $\frac{a}{0}=\infty$ if $a>0$ and 0 if $a=0$ ). Hence

$$
E(\mu)=\bigcup_{1 \leq i \leq j \leq n}\left[y_{j}-k_{i}-\ldots-k_{j}, y_{i}+k_{i}+\ldots+k_{j}\right],
$$

(where $[a, b]=\varnothing$ if $b<a)$.

Proof. Since the right hand side of (2.5) is larger than or equal to $1 / 2$ if and only if at least one of the terms in max is, (2.6) follows easily from (2.5). To prove (2.5) we fix $x \in \mathbb{R}$ and for $h>0$ we find $i \leq j$ such that $[x-h, x+h] \cap\left\{y_{1}, \ldots, y_{n}\right\}=\left\{y_{i}, \ldots, y_{j}\right\}$. Then $h \geq \max \left\{x-y_{i}, y_{j}-x\right\}$ and so by (1.5) $M \mu(x)$ is less than or equal to the right hand side of (2.5). On the other hand if $i \leq j$ and $2 x<y_{i}+y_{j}$, then by taking $h=y_{j}-x>0$ we see that $\frac{k_{i}+\ldots+k_{j}}{\left|2 x-y_{i}-y_{j}\right|+y_{j}-y_{i}}=\frac{k_{i}+\ldots+k_{j}}{2\left(y_{j}-x\right)} \leq M \mu(x)$ and similarly if $2 x>y_{i}+y_{j}$. Finally if $x=y_{i}=y_{j}$ then both sides of (2.5) are equal to $\infty$ (if $k_{i}>0$ ) or to 0 (if $k_{i}=0$ ). Hence (2.5) holds.

In general the set $E(\mu)$ is hard to analyze. But the next lemma which is proved in [1] shows that it is sufficient to assume that $E(\mu)$ is an interval. For completeness we include a proof here.

Lemma 2. Let $\mu$ be any measure of the form (2.1). Then there exists a measure $\mu^{*}$ of the same form, having at most as many positions as $\mu$ and such that $E\left(\mu^{*}\right)$ is connected and $R(\mu) \leq R\left(\mu^{*}\right)$.

Proof. Let $\mu$ be written in reduced form. If $E(\mu)$ is not connected let $(a, b)$ be a bounded component of its complement where $a<b$. Then by (2.6) we must have $a=y_{i}+k_{i}+\ldots+k_{r}$ and $b=y_{j}-k_{l}-\ldots-k_{j}$ for some $i<j, r \geq i, l \leq j$ and since $(a, b) \cap \bigcup_{s=1}^{n}\left[y_{s}-k_{s}, y_{s}+k_{s}\right]=\varnothing$ there is an $s \in\{i, \ldots, j-1\}$ such that $y_{s}+k_{s} \leq a<b \leq y_{s+1}-k_{s+1}$. Let $y_{p}^{\prime}=y_{p}+b-a$ for $p \leq s$ and $y_{p}^{\prime}=y_{p}$ for $p>s$. Then for $\mu^{\prime}=\sum_{p=1}^{n} k_{p} \delta_{y_{p}^{\prime}}$, using Lemma 1 it is easy to see that $R(\mu) \leq R\left(\mu^{\prime}\right)$ and 
moreover that the complement of $E\left(\mu^{\prime}\right)$ has at least one bounded component less than the complement of $E(\mu)$. By repeating the above process one can now easily complete the proof.

From now on we will, unless specified otherwise, consider only measures $\mu$ such that $E(\mu)$ is an interval. It has also been proved in [1] that we can further reduce the problem to considering only measures that satisfy a certain separability condition as the following lemma shows (for which we give a different proof).

Lemma 3. It is sufficient to consider measures $\mu$ of the form (2.1) with $E(\mu)$ connected that also satisfy the separability condition

$$
y_{i+1}-y_{i}>k_{i}+k_{i+1}
$$

for $i=1, \ldots, n-1$.

Proof. Suppose that $y_{i+1}-y_{i} \leq k_{i}+k_{i+1}$ for some $i$. Then choose any $z \in$ $\left[y_{i+1}-k_{i}, y_{i}+k_{i+1}\right] \cap\left[y_{i}, y_{i+1}\right] \neq \varnothing$ and define $\mu^{\prime}=\mu-k_{i} \delta_{y_{i}}-k_{i+1} \delta_{y_{i+1}}+\left(k_{i}+k_{i+1}\right) \delta_{z}$. Then $\mu^{\prime}$ has at most $n-1$ positions, $N\left(\mu^{\prime}\right)=N(\mu)$ and using Lemma 1 one can see that $E(\mu) \subset E\left(\mu^{\prime}\right)$. Indeed the families of intervals that by Lemma 1 constitute the sets $E(\mu)$ and $E\left(\mu^{\prime}\right)$ respectively may differ only in the intervals that involve $y_{i}$ or $y_{i+1}$. However the interval $\left[y_{i}-k_{l}-\ldots-k_{i}, y_{l}+k_{l}+\ldots+k_{i}\right], l<i$ in $E(\mu)$ is transformed into the interval $\left[z-k_{l}-\ldots-k_{i}-k_{i+1}, y_{l}+k_{l}+\ldots+k_{i}+k_{i+1}\right]$ in $E\left(\mu^{\prime}\right)$ and so since $z \leq y_{i}+k_{i+1}$ it doesn't get smaller. One can similarly check all other cases of such intervals to conclude that $E(\mu) \subset E\left(\mu^{\prime}\right)$. Apply now the procedure of Lemma 2 to $\mu^{\prime}$ to obtain a measure $\mu^{\prime \prime}$ with fewer positions than $\mu$, with $E\left(\mu^{\prime \prime}\right)$ connected and such that $R(\mu) \leq R\left(\mu^{\prime \prime}\right)$.

Repeating now the whole process, whenever possible, and observing that at each stage we reduce the number of positions by at least one it is easy to complete the proof of the lemma.

Therefore from now on we will consider only measures $\mu$ in reduced form (2.1) for which $E(\mu)$ is an interval and that satisfy the inequalities (2.7). We will call any such measure admissible. For these measures we have the following (see also [1]).

Lemma 4. If the measure $\mu$ given in reduced form by (2.1) is admissible, then

$$
E(\mu)=\left[y_{1}-k_{1}, y_{n}+k_{n}\right] .
$$

Proof. Adding inequalities from (2.7) we obtain

$$
y_{j}-y_{i}>k_{i}+2 k_{i+1}+\ldots+2 k_{j-1}+k_{j}
$$

whenever $i<j$. In particular for any $j>1$ we have $y_{j}-k_{1}-. .-k_{j}>y_{1}+$ $k_{2}+\ldots+k_{j-1} \geq y_{1}$ and similarly for every $i<n, y_{i}+k_{i}+\ldots+k_{n}<y_{n}$. Now from (2.6), the assumption that $E(\mu)$ is an interval and since $\left[y_{1}-k_{1}, y_{1}+k_{1}\right] \cup$ $\left[y_{n}-k_{n}, y_{n}+k_{n}\right] \subset E(\mu)$, 2.8) follows.

Now let $\mu$ be an admissible measure with reduced form (2.1). For any $1 \leq i \leq$ $j \leq n$ we define the interval $([a, b]=\varnothing$ if $b<a)$

$$
I_{i, j}=I_{i, j}(\mu)=\left[y_{j}-k_{i}-\ldots-k_{j}, y_{i}+k_{i}+\ldots+k_{j}\right] .
$$

Therefore by Lemmas 1 and 4 , the nonempty of the intervals $I_{i, j}$ 's form a covering of the interval $E(\mu)=\left[y_{1}-k_{1}, y_{n}+k_{n}\right]$. 


\section{THE "GAP" INTERVAL}

Now let $\mu$ be an admissible measure written in reduced form as in (2.1). We will associate to $\mu$ an interval $J(\mu)$ that will give a more geometric understanding of the covering properties of the $I_{i, j}$ 's.

First we define the gaps of $\mu$ as the positive numbers

$$
x_{i}=y_{i+1}-y_{i}-k_{i+1}-k_{i}
$$

for $1 \leq i \leq n$. The $x_{i}$ 's are the lengths of the gaps between the intervals $I_{i, i}=$ $\left[y_{i}-k_{i}, y_{i}+k_{i}\right], 1 \leq i \leq n$, and so correspond to the part of the interval $E(\mu)$ that has to be covered by intervals of the form $I_{i, j}$ where $i<j$.

Now we define the points

$$
a_{1}=0, a_{2}=x_{1}, a_{3}=x_{1}+x_{2}, \ldots, a_{n}=x_{1}+\ldots+x_{n-1}
$$

and the gap interval of $\mu$ as the interval

$$
J(\mu)=\left[a_{1}, a_{n}\right] .
$$

The gap interval can be obtained from $E(\mu)$ by collapsing the intervals $I_{i, i}=$ $\left[y_{i}-k_{i}, y_{i}+k_{i}\right], 1 \leq i \leq n$, to the points $a_{i}$. This can be described by defining the (measure preserving and discontinuous) mapping

$$
Q=Q_{\mu}: J(\mu) \rightarrow E(\mu) \backslash\left(I_{1,1} \cup \ldots \cup I_{n, n}\right)
$$

that satisfies $Q(x)=y_{i}+k_{i}+\left(x-a_{i}\right)$ whenever $x \in\left(a_{i}, a_{i+1}\right), 1 \leq i<n$. Thus $Q$ maps each subinterval $\left(a_{i}, a_{i+1}\right)$ of $J(\mu)$ onto the corresponding gap $\left(y_{i}+k_{i}, y_{i+1}-\right.$ $\left.k_{i+1}\right)$ of $E(\mu) \backslash\left(I_{1,1} \cup \ldots \cup I_{n, n}\right)$.

It will be important to also consider the intervals

$$
J_{i}=\left[a_{i}-k_{i}, a_{i}+k_{i}\right]
$$

around each of the points $a_{i}, 1 \leq i \leq n$, of $J(\mu)$. Let

$$
\mathcal{F}(\mu)=\left\{J_{1}, \ldots, J_{n}\right\}
$$

denote the corresponding family of all these intervals and let

$$
J_{i}^{+}=\left[a_{i}, a_{i}+k_{i}\right] \text { and } J_{i}^{-}=\left[a_{i}-k_{i}, a_{i}\right]
$$

denote the right and left half of $J_{i}$ respectively.

Observing now that $y_{n}-y_{1}=x_{1}+\ldots+x_{n-1}+k_{1}+2 k_{2}+\ldots+2 k_{n-1}+k_{n}$ and that $\left|J_{1}\right|+\ldots+\left|J_{n}\right|=2 N(\mu)$ we conclude that $|E(\mu)|=|J(\mu)|+2 N(\mu)$ and so

$$
R(\mu)=\frac{|J(\mu)|}{\left|J_{1}\right|+\ldots+\left|J_{n}\right|}+1 .
$$

The close relation between the family $\mathcal{F}(\mu)$ and the covering properties of the $I_{i, j}$ 's can be seen from the following.

Lemma 5. Let $1 \leq i<j \leq n$. Then $I_{i, j} \neq \varnothing$ if and only if $J_{i} \cap J_{j} \neq \varnothing$ in which case we also have $I_{i, j} \backslash\left(I_{1,1} \cup \ldots \cup I_{n, n}\right) \subseteq Q\left(J_{i} \cup J_{j}\right)$. Moreover if $a_{j} \notin J_{i}^{+}$and $a_{i} \notin J_{j}^{-}$then $\left|I_{i, j}\right|=\left|J_{i}^{+} \cap J_{j}^{-}\right|$.

Proof. Clearly $I_{i, j} \neq \varnothing$ if and only if $y_{j}-y_{i} \leq 2\left(k_{i}+\ldots+k_{j}\right)$. Intoducing the $x_{r}$ 's we can write this as $a_{j}-a_{i}=x_{i}+\ldots+x_{j} \leq k_{i}+k_{j}$ or $a_{j}-k_{j} \leq a_{i}+k_{i}$ which exactly means that $J_{i} \cap J_{j} \neq \varnothing$. On the other hand if $Q(x) \in I_{i, j}$ then it is easy to see, using the separability condition (2.7]) as in the proof of Lemma 4, that we must have $x \in\left(a_{t}, a_{t+1}\right)$ for some $t$ with $i \leq t<t+1 \leq j$. Hence $I_{i, j} \subseteq Q\left(J_{i} \cup J_{j}\right)$ since 
$J_{i} \cap J_{j} \neq \varnothing$. The last statement follows since $\left|I_{i, j}\right|=2\left(k_{i}+\ldots+k_{j}\right)-\left(y_{j}-y_{i}\right)=$ $\left(a_{i}+k_{i}\right)-\left(a_{j}-k_{j}\right)$ when $I_{i, j} \neq \varnothing$.

An immediate consequence of this lemma is the following.

Proposition 1. We have $J(\mu) \subseteq J_{1} \cup \ldots \cup J_{n}$, hence the family $\mathcal{F}(\mu)$ is a covering of the interval $J(\mu)$. In particular $C \leq 2$.

Proof. Since $E(\mu)$ is connected, given any $r$ with $1 \leq r<n$ there exist $i<j$ such that the interval $I_{i, j} \neq \varnothing$ covers points from $\left(y_{r}+k_{r}, y_{r+1}-k_{r+1}\right)$. It follows then easily that $i \leq r \leq j$ and since by the previous lemma $J_{i} \cap J_{j} \neq \varnothing$ we conclude that $\left[a_{r}, a_{r+1}\right] \subseteq J_{i} \cup J_{j}$. Hence $J(\mu) \subseteq J_{1} \cup \ldots \cup J_{n}$ and in particular $|J(\mu)| \leq\left|J_{1}\right|+\ldots+\left|J_{n}\right|$. So in view of (3.8) we have $R(\mu) \leq 2$ which completes the proof.

To reduce the constant 2 in the previous proposition we will have to study more closely the overlapping of the covering $\mathcal{F}(\mu)$ that is forced by the requirement that the $I_{i, j}$ 's cover the interval $E(\mu)$. In this direction we have the following.

Lemma 6. If $i<r$ and $a_{r} \in J_{i}^{+}$then

$$
I_{i, i} \cup \ldots \cup I_{i, r}=\left[y_{i}-k_{i}, y_{i}+k_{i}+\ldots+k_{r}\right]
$$

Similarly if $l<i$ and $a_{l} \in J_{i}^{-}$then

$$
I_{l, i} \cup \ldots \cup I_{i, i}=\left[y_{i}-k_{l}-\ldots-k_{i}, y_{i}+k_{i}\right] .
$$

Proof. For any interval $I$ let $l(I)$ and $r(I)$ denote its left and its right endpoints respectively. It is easy to see that $a_{r} \in J_{i}^{+}$is equivalent to $y_{r}-y_{i} \leq 2 k_{i}+\ldots+$ $2 k_{r-1}+k_{r}$. Now for any $s \geq i$ let $h_{s}=2 k_{i}+\ldots+2 k_{s-1}+k_{s}-\left(y_{s}-y_{i}\right)$. Then of course $h_{s+1}-h_{s}=k_{s+1}+k_{s}-\left(y_{s+1}-y_{s}\right)<0$ by (2.7). Since we have assumed that $h_{r} \geq 0$ we have $h_{i}>h_{i+1}>\ldots>h_{r} \geq 0$. Therefore for $i \leq s \leq r, I_{i, s} \neq \varnothing$ for $i \leq s \leq r$, and if $i \leq s<r$ we have $l\left(I_{i, s+1}\right) \leq r\left(I_{i, s}\right)$ since their difference is $-h_{s+1}$. This obviously implies (3.9) and (3.10) can be proved in the same way.

Therefore defining (for any fixed $i$ ),

$$
\begin{aligned}
l_{i} & =\min \left\{l \leq i: a_{l} \in J_{i}^{-}\right\} \\
& =\min \left\{l \leq i: y_{i}-y_{l} \leq k_{l}+2 k_{l+1}+\ldots+2 k_{i}\right\}, \\
r_{i} & =\max \left\{r \geq i: a_{r} \in J_{i}^{+}\right\} \\
& =\max \left\{\left\{r \geq i: y_{r}-y_{i} \leq 2 k_{i}+\ldots+2 k_{r-1}+k_{r}\right\}\right.
\end{aligned}
$$

the previous lemma implies that

$$
F_{i}=\left[y_{i}-k_{l_{i}}-\ldots-k_{i}, y_{i}+k_{i}+\ldots+k_{r_{i}}\right]=I_{l_{i}, i} \cup \ldots \cup I_{i, i} \cup \ldots \cup I_{i, r_{i}},
$$

where the above equation defines $F_{i}$. We also have the following.

Lemma 7. Let $1 \leq i \leq n$ be fixed. Then the nonempty of the closed intervals $I_{0, i}, \ldots, I_{l_{i}-1, i}, I_{i, r_{i}+1}, \ldots, I_{i, n+1}$ (if any) are pairwise disjoint and each of them is disjoint from $F_{i}$.

Proof. Let $h_{s}$ for $s \geq i$ be as in the proof of Lemma 6. If $r_{i} \leq p<q \leq n+1$ and $I_{i, p}, I_{i, q} \neq \varnothing$ then $l\left(I_{i, q}\right)-r\left(I_{i, p}\right)=-h_{p+1}-\ldots-h_{q}>0$ since we have seen that $h_{r_{i}+1} \geq h_{p+1}>h_{p+2}>\ldots>h_{q}$ and by the definition of $r_{i}$ we have $h_{r_{i}+1}<0$. Hence $I_{i, p} \cap I_{i, q}=\varnothing$. This easily implies the lemma for $F_{i}$ and the nonempty (if any) of the intervals $I_{i, r_{i}+1}, \ldots, I_{i, n+1}$. The proof for the other intervals is similar. 
Remark. From the previous lemmas we conclude that the intervals $I_{i, j}, 1 \leq i \leq j \leq$ $n$, cover the interval $E(\mu)$ by forming the main intervals $F_{s}, 1 \leq s \leq n$, and possibly by some intervals $I_{p, q}$ such that $a_{q} \notin J_{p}^{+}$and $a_{p} \notin J_{q}^{-}$with $\left|I_{p, q}\right|=\left|J_{p}^{+} \cap J_{q}^{-}\right|$. We will call intervals like $I_{p, q}$, if any, special intervals of the covering $\left\{I_{i, j}\right\}$.

\section{THE LOWER BOUND}

To obtain the lower bound it would be convenient to also consider the following modified "norm"

$$
N^{*}(\mu)=k_{0}+2 k_{1}+\ldots+2 k_{n}+k_{n+1} .
$$

The corresponding modified ratio of $\mu$ is defined by

$$
R^{*}(\mu)=\frac{|E(\mu)|-k_{0}-k_{n+1}}{N^{*}(\mu)}=\frac{y_{n+1}-y_{0}}{k_{0}+2 k_{1}+\ldots+2 k_{n}+k_{n+1}}
$$

where $\mu$ is any admissible measure written in reduced form as $\mu=\sum_{i=0}^{n+1} k_{i} \delta_{y_{i}}$ (note that we have changed the range of summation here). It is easy to see that $R^{*}(\mu)>R(\mu)>1$ for any admissible $\mu$. However we also have the following.

Lemma 8. For any admissible measure $\mu$ and every $\varepsilon>0$ there exists a measure $\widetilde{\mu}$ such that

$$
R(\widetilde{\mu}) \geq R^{*}(\mu)-\varepsilon
$$

Therefore $R^{*}(\mu) \leq C$ for any such $\mu$.

Proof. Let

$$
\mu_{1}=\sum_{i=0}^{n} k_{i} \delta_{y_{i}}+k_{n+1} \delta_{y_{n+1}}+\sum_{i=1}^{n} k_{n+1-i} \delta_{2 y_{n+1}-y_{n+1-i}}
$$

be obtained by reflection with respect to the last position of $\mu$ (dropping the term $k_{0} \delta_{2 y_{n+1}-y_{0}}$ ) and for any $p \geq 1$ let $\mu_{p+1}$ be obtained from $\mu_{p}$ by a translation of $2\left(y_{n+1}-y_{0}\right)$. Then for any integer $P>1$ it is easy to see that the measure $\nu_{P}=\mu_{1}+\ldots+\mu_{P}+k_{0} \delta_{2 P\left(y_{n+1}-y_{0}\right)+y_{0}}$ is admissible and hence satisfies $\left|E\left(\nu_{P}\right)\right|=$ $2 k_{0}+2 P\left(y_{n+1}-y_{0}\right)=2 k_{0}+2 P\left(|E(\mu)|-k_{0}-k_{n+1}\right)$ (by Lemma 4) and $N\left(\nu_{P}\right)=$ $k_{0}+N^{*}(\mu) P$. Therefore as $P \rightarrow \infty$ we have $R\left(\nu_{P}\right) \rightarrow R^{*}(\mu)$. This completes the proof of the lemma.

Therefore it would be sufficient to study the modified ratio $R^{*}$.

To describe our construction we consider now any measure $\nu$ that satisfies the separability condition (2.7). We do not assume that $E(\nu)$ is connected. Writting $\nu$ in reduced form as $\sum_{i=1}^{n} k_{i} \delta_{y_{i}}$ we fix integers $1 \leq s, t \leq n$ and define the measure

$$
T_{s, t} \nu=k_{0} \delta_{y_{0}}+\nu+k_{n+1} y_{n+1}
$$

where

$$
y_{0}=2 y_{1}-y_{s}-2 k_{1}-k_{s}, k_{0}=y_{s}-y_{1}-k_{2}-\ldots-k_{s-1}
$$

and

$$
y_{n+1}=2 y_{n}-y_{t}+2 k_{n}+k_{t}, k_{n+1}=y_{n}-y_{t}-k_{t+1}-\ldots-k_{n-1} .
$$

Then we have the following.

Lemma 9. $E\left(T_{s, t} \nu\right)$ doesn't have more gaps than $E(\nu)$. That is the added intervals $\left(y_{0}, y_{1}-k_{1}\right),\left(y_{n}+k_{n}, y_{n+1}\right)$ are contained in $E\left(T_{s, t} \nu\right)$. 
Proof. We will give the proof only for the interval $\left(y_{0}, y_{1}-k_{1}\right)$, the other case being similar. It is easy to see that (4.6) implies that $y_{s}-y_{0} \leq 2 k_{0}+\ldots+2 k_{s-1}+k_{s}$ hence in view of Lemma 6 we have $I_{0,0}(\nu) \cup I_{0,1}(\nu) \cup \ldots \cup I_{0, s}(\nu)=\left[y_{0}-k_{0}, y_{0}+k_{0}+\ldots+k_{s}\right]$. But we also have from (4.6) that $y_{0}+k_{0}+\ldots+k_{s}=y_{1}-k_{1}$ and this completes the proof.

Hence the operation $T_{s, t}$ does not create any new gaps. However we have the advantage of using the special interval $I_{0, n+1}$, which will be nonempty if $s>t$ (see Lemma 5), to possibly cover gaps of our initial set $E(\nu)$. For this purpose we argue as follows.

Let $\mu$ be any, admissible now, measure written in reduced form as $\mu=\sum_{i=1}^{m} k_{i}^{\prime} \delta_{z_{i}}$ where for simpicity we assume that $z_{1}=0$. Fix now two positive real numbers $A, \alpha>0$ and consider the scaled measure $\alpha . \mu$ defined by

$$
\alpha . \mu=\sum_{i=1}^{m} \alpha k_{i}^{\prime} \delta_{\alpha z_{i}} .
$$

Since we have scaled both the $k_{i}^{\prime}$ 's and the $z_{i}^{\prime}$ 's the measure $\alpha . \mu$ is also admissible and so the measure

$$
\nu=\mu+\operatorname{trasl}_{A}(\alpha . \mu)=\sum_{i=1}^{m} k_{i}^{\prime} \delta_{z_{i}}+\sum_{i=1}^{m} \alpha k_{i}^{\prime} \delta_{\alpha z_{i}+A}=\sum_{i=1}^{2 m} k_{i} \delta_{y_{i}}
$$

satisfies the separability condition (2.7) as long as $A>k_{m}^{\prime}+\alpha k_{1}^{\prime}$. We will next take in (4.5), with $n=2 m$, as $s$ the last position of $\mu$, so $s=2 m$, and as $t$ the first position of the translated $\alpha . \mu$, so $t=m+1$ and consider the measure

$$
T \mu=T_{2 m, m+1} \nu .
$$

We now have the following.

Lemma 10. Choosing

$$
\alpha=2 R(\mu) \text { and } A=\left(\alpha^{2}-\alpha\right) N(\mu)+(\alpha-1) k_{1}^{\prime}
$$

the measure $T \mu$ will be admissible (hence $E(T \mu)$ is connected) and moreover

$$
R^{*}(T \mu)=\frac{20 R(\mu)^{2}-4 R(\mu)}{12 R(\mu)^{2}-2 R(\mu)+1} .
$$

Proof. We first show that $E(T \mu)$ is connected. Using (4.6), (4.7) and (4.9) it is easy to see that if $T \mu=\sum_{i=0}^{n+1} k_{i} \delta_{y_{i}}$, then $y_{0}=-\alpha z_{m}-A-2 k_{1}^{\prime}-\alpha k_{m}^{\prime}$, $k_{0}=\alpha z_{m}+A-(1+\alpha) N(\mu)+k_{1}^{\prime}+\alpha k_{m}^{\prime}, y_{n+1}=2 \alpha z_{m}+A+\alpha k_{1}^{\prime}+2 \alpha k_{m}^{\prime}$ and $k_{n+1}=\alpha z_{m}-\alpha N(\mu)+\alpha k_{1}^{\prime}+\alpha k_{m}^{\prime}$. Since $E(\mu)$ and $E(\alpha . \mu)$ are connected Lemma 9 implies that $E(T \mu)$ would be connected as long as the special interval $I_{0, n+1}$ covers the possible gap $\left(z_{m}+k_{m}^{\prime}, A-\alpha k_{1}^{\prime}\right)$ of $E(\nu)$. We have $k_{0}+\ldots+k_{n+1}=$ $2 \alpha z_{m}+A-\alpha N(\mu)+(1+\alpha) k_{1}^{\prime}+2 \alpha k_{m}^{\prime}$, and so (2.10), Lemma 4 and (4.11) imply that $l\left(I_{0, n+1}\right)=y_{n+1}-k_{0}-\ldots-k_{n+1}=\alpha N(\mu)-k_{1}^{\prime}=|E(\mu)|-k_{1}^{\prime}=z_{m}+k_{m}^{\prime}$ and $r\left(I_{0, n+1}\right)=y_{0}+k_{0}+\ldots+k_{n+1}=\alpha z_{m}-\alpha N(\mu)+(\alpha-1) k_{1}^{\prime}+\alpha k_{m}^{\prime}=$ $\left(\alpha^{2}-\alpha\right) N(\mu)-k_{1}^{\prime}=A-\alpha k_{1}^{\prime}$. Hence $E(T \mu)$ is connected. It is now easy to compute $N^{*}(T \mu)=\left(3 \alpha^{2}-\alpha+1\right) N(\mu)$ and $|E(T \mu)|-k_{0}-k_{n+1}=y_{n+1}-y_{0}=$ $\left(5 \alpha^{2}-2 \alpha\right) N(\mu)$ from which (4.12) follows.

Using the previous lemma we can now give the following lower bound for $C$. 
Proposition 2. The best constant $C$ in the weak $(1,1)$ inequality for the centered Hardy-Littlewood maximal operator satisfies

$$
C \geq \frac{11+\sqrt{61}}{12}=1.5675208 \ldots
$$

Proof. In view of Lemma 8 and (4.12) we have

$$
\frac{20 R(\mu)^{2}-4 R(\mu)}{12 R(\mu)^{2}-2 R(\mu)+1} \leq C
$$

for any admissible measure $\mu$. Choosing a sequence of admissible measures $\mu_{q}$, $q \geq 1$ such that $R\left(\mu_{q}\right) \rightarrow C$ as $q \rightarrow \infty$ we conclude, using (4.14) that

$$
12 C^{2}-22 C+5 \geq 0 .
$$

But now (4.13) follows from (4.15) since $C>1$.

Remarks. 1) The above proof can be adapted to provide examples of measures $\mu$ with $R(\mu)$ as close as we want to $1.5675208 \ldots$. Indeed let $f(x)=\frac{20 x^{2}-4 x}{12 x^{2}-2 x+1}$. Take for example $\mu_{1}=\delta_{0}+\delta_{3}$ and having defined $\mu_{p}$ consider $T \mu_{p}$ and apply the procedure in Lemma 8 to obtain a measure $\mu_{p+1}$ such that $R\left(\mu_{p+1}\right) \geq f\left(R\left(\mu_{p}\right)\right)-\varepsilon_{p}$ where $\varepsilon_{p}>0$. Choosing the $\varepsilon_{p}$ 's to tend to 0 sufficiently fast we will have $R\left(\mu_{p}\right) \rightarrow$ $1.5675208 \ldots$ as $p \rightarrow \infty$. However after the first few steps the measures $\mu_{p}$ will be rather complicated.

2) There are many other constuctions like $T \mu$ that lead to certain quadratic equations similar to (4.15) and so to lower bounds for $C$. However the above constuction gives the best lower bound for $C$ as far as we know.

\section{THE UPPER BOUND}

To obtain the upper bound for $C$ claimed in Theorem 1 we will first introduce some more notation. Fix an admissible measure $\mu$ written in reduced form as in (2.1) and let its gap interval $J(\mu)$ and its corresponding cover (by Proposition 1) $\mathcal{F}(\mu)=\left\{J_{1}, \ldots, J_{n}\right\}$ be as in Section 3. For any family $\mathcal{G}$ of closed intervals that covers the interval $J(\mu)$ we consider the set

$$
e_{1}(\mathcal{G})=\{x \in J(\mu): x \text { belongs to exactly one interval of } \mathcal{G}\}
$$

associated to $\mathcal{G}$ and its relative measure

$$
\lambda(\mathcal{G})=\frac{\left|e_{1}(\mathcal{G})\right|}{|J(\mu)|} .
$$

We will say that $\mathcal{G}$ is generated by $\mathcal{F}(\mu)$ if it can be constucted by moving some subintervals of $J_{1}, \ldots, J_{n}$ to other parts of $J(\mu)$ in such a way that the resulting family $\mathcal{G}$ (which will in general contain more intervals than $\mathcal{F}(\mu)$ ) still covers $J(\mu)$. Obviously

$$
\sum_{I \in \mathcal{G}}|I|=\left|J_{1}\right|+\ldots+\left|J_{n}\right|
$$

whenever $\mathcal{G}$ is generated by $\mathcal{F}(\mu)$.

We now have the following. 
Lemma 11. For every cover $\mathcal{G}$ generated by $\mathcal{F}(\mu)$ the following inequality holds:

$$
R(\mu) \leq \frac{3-\lambda(\mathcal{G})}{2-\lambda(\mathcal{G})}
$$

Proof. Since points in $J(\mu) \backslash e_{1}(\mathcal{G})$ are covered at least twice by the intervals of $\mathcal{G}$ we have $\sum_{I \in \mathcal{G}}|I| \geq\left|e_{1}(\mathcal{G})\right|+2\left(|J(\mu)|-\left|e_{1}(\mathcal{G})\right|\right)=(2-\lambda(\mathcal{G}))|J(\mu)|$. Hence (5.4) follows from (5.3) combined with (3.8).

Remark. This lemma in a sense explains why there are measures with $R(\mu)>\frac{3}{2}$. Thus in order to make $R(\mu)$ big one must first have a relatively large $e_{1}(\mathcal{F}(\mu))$ but also be careful not to introduce a lot of overlapping in $\mathcal{F}(\mu)$ that could produce a cover $\mathcal{G}$ with $\lambda(\mathcal{G})$ small.

It follows from the previous lemma that to obtain upper bounds for $R(\mu)$ and hence for $C$ it is sufficient to generate good covers of $J(\mu)$ by displacing some subintervals of the intervals of $\mathcal{F}(\mu)$. One way to do this is through the following.

Lemma 12. For any admissible measure $\mu$ there exists a cover $\mathcal{G}$ of $J(\mu)$ generated by $\mathcal{F}(\mu)$ such that

$$
e_{1}(\mathcal{G}) \subseteq J(\mu) \backslash e_{1}(\mathcal{F}(\mu))
$$

In particular

$$
\lambda(\mathcal{G}) \leq 1-\lambda(\mathcal{F}(\mu)) .
$$

Proof. The basic observation is that given $x \in e_{1}\left(\mathcal{F}(\mu)\right.$ ) with say $x \in J_{i}$ (for a unique $i$ ) then $Q(x) \in E(\mu)$ must be covered by some interval $I_{i, r}$ with $r>i$ such that $a_{i} \notin J_{r}^{-}$if $x>a_{i}$ or by some interval $I_{l, i}$ with $l<i$ such that $a_{i} \notin J_{l}^{+}$if $x<a_{i}$. Indeed if $Q(x) \in I_{p, q}$ then $p<q$ and so Lemma 5 and its proof imply that $x \in J_{p} \cup J_{q}$ and $x \in\left(a_{t}, a_{t+1}\right)$ for some $t$ with $p \leq t<t+1 \leq q$. Therefore the uniqueness of $i$ implies that either $p=i$ and $x \notin J_{q}^{-}$or $q=i$ and $x \notin J_{p}^{+}$.

For $i$ fixed, let $s \geq i$ be the largest integer such that $a_{i} \in J_{s}^{-}$. Then points of $Q\left(J_{i}^{+} \cap e_{1}(\mathcal{F}(\mu))\right)$ would be covered by $F_{i}$ only if $a_{s} \in J_{i}^{+}$and using the above observation they will be located to the right of the interval $I_{i, i} \cup \ldots \cup I_{i, s}$ that has by Lemma 6 length $k_{i}+\ldots+k_{s}$. Hence, using again Lemma 6 , the part of $Q\left(J_{i}^{+} \cap e_{1}(\mathcal{F}(\mu))\right)$ covered by $F_{i}$ (if any) has measure at most $k_{s+1}+\ldots+k_{r_{i}}=$ $\left|J_{s+1}^{-}\right|+\ldots+\left|J_{r_{i}}^{-}\right| \leq\left|J_{i}^{+} \cap J_{i+1}^{-}\right|+\ldots+\left|J_{i}^{+} \cap J_{r_{i}}^{-}\right|$(where $r_{i}$ is defined by (3.11) by the definition of $s$. Also by Lemma 5 the part of $Q\left(J_{i}^{+} \cap e_{1}(\mathcal{F}(\mu))\right)$ covered by any special interval $I_{i, r}$ (if any) for any $r>r_{i}$ has measure at most $\left|J_{i}^{+} \cap J_{r}^{-}\right|$. It follows then easily that we can cover $J_{i}^{+} \cap e_{1}(\mathcal{F}(\mu))$ by appropriately placing certain subintervals of $J_{i}^{+} \cap J_{i+1}^{-}, \ldots, J_{i}^{+} \cap J_{n}^{-}$over it and moreover after we have done this we will still have a cover of $J(\mu)$ since the displaced subintervals are initially also covered by $J_{i}^{+}$which has not been affected. Then every point of $J_{i}^{+} \cap e_{1}(\mathcal{F}(\mu))$ will be covered at least twice and so wouldn't belong to $e_{1}$ of the new cover. We apply this procedure for all $i$ 's such that $J_{i}^{+} \cap e_{1}(\mathcal{F}(\mu)) \neq \varnothing$. We wouldn't be tampering with the same $J_{r}^{-}$more than once since Lemma 5 easily implies that $I_{i, r}=\varnothing$ whenever $J_{i}^{+} \cap e_{1}(\mathcal{F}(\mu)) \neq \varnothing, J_{p}^{+} \cap e_{1}(\mathcal{F}(\mu)) \neq \varnothing$ and $i<p<r$.

Now we apply an analogous procedure for all $j$ 's such that $J_{j}^{-} \cap e_{1}(\mathcal{F}(\mu)) \neq \varnothing$ using the same remarks as before. Doing this we might tamper with some $J_{i}^{+}$such that $J_{i}^{+} \cap e_{1}(\mathcal{F}(\mu)) \neq \varnothing$. However if $i<j, J_{i}^{+} \cap e_{1}(\mathcal{F}(\mu)) \neq \varnothing$ and $J_{j}^{-} \cap e_{1}(\mathcal{F}(\mu)) \neq$ 
$\varnothing$ then the interval $I_{i, j}$ would be special and so by Lemma $5\left|I_{i, j}\right|=\left|J_{i}^{+} \cap J_{j}^{-}\right|$. Since obviously $J_{i}^{+} \cap J_{j}^{-}$does not meet $e_{1}(\mathcal{F}(\mu))$ it follows that $I_{i, j}$ can cover points in at most one of the sets $Q\left(J_{i}^{+} \cap e_{1}(\mathcal{F}(\mu))\right)$ and $Q\left(J_{j}^{-} \cap e_{1}(\mathcal{F}(\mu))\right)$. Hence we would never tamper with both $J_{i}^{+}$and $J_{j}^{-}$for any such $i, j$. Therefore after the whole procedure is over we obtain a family $\mathcal{G}$ that still covers $J(\mu)$, is generated by $\mathcal{F}(\mu)$, and has the property that each $x \in e_{1}(\mathcal{F}(\mu))$ is covered by at least two distinct intervals of $\mathcal{G}$. This clearly implies (5.5) and so completes the proof.

This lemma now implies our upper bound for $C$.

Proposition 3. The best constant $C$ in the weak $(1,1)$ inequality for the centered Hardy-Littlewood maximal operator satisfies

$$
C \leq \frac{5}{3}
$$

Proof. Let $\mu$ be any admissible measure. If $\lambda(\mathcal{F}(\mu)) \leq \frac{1}{2}$ then (5.4) with $\mathcal{G}=\mathcal{F}(\mu)$ implies that $R(\mu) \leq \frac{5}{3}$. On the other hand if $\lambda(\mathcal{F}(\mu))>\frac{1}{2}$ then Lemma 12 provides a cover $\mathcal{G}$ of $J(\mu)$ that is generated by $\mathcal{F}(\mu)$ and satisfies $\lambda(\mathcal{G}) \leq 1-\lambda(\mathcal{F}(\mu))<\frac{1}{2}$. Then (5.4) implies that $R(\mu)<\frac{5}{3}$. This completes the proof.

\section{REFERENCES}

1. J.M.Aldaz. Remarks on the Hardy-Littlewood maximal function, Proc. of the Royal Society of Edinburgh 128A (1998), no.1, 1-9. MR 99b:42020

2. D.A.Brannan, W.K. Hayman. Research problems in complex analysis, Bull. London Math. Soc. 21 (1989), 1-35. MR 89m:30001

3. A.Bernal. A note on the one-dimensional maximal function, Proc. of the Royal Society of Edinburgh 111A (1989), 325-328. MR 90i:28010

4. H.Carlsson. A new proof of the Hardy Littlewood maximal theorem, Bull. London Math. Soc. 16 (1984), 595-596. MR 86g:42024

5. A.M.Garsia. Topics in almost everywhere convergence, Markkham Publishing Company, Chicago, 1970. MR 41:5869

6. L.Grafakos, S.Montgomery-Smith. Best constants for uncentered maximal functions, Bull. London Math. Soc. 29 (1997), no.1, 60-64. MR 98b:42031

7. M. de Guzmán. Real variable methods in Fourier analysis, North-Holland Mathematical Studies 46. Notas de Mathemática (75), 1981. MR 83j:42019

8. J. Manfredi, F. Soria. On a dynamical system relared to estimating the best constant in an inequality of Hardy and Littlewood, unpublished manuscript.

9. M.Trinidad Menarguez, F.Soria. Weak type $(1,1)$ inequalities of maximal convolution operators, Rendiconti del Circolo Mathematico di Palermo (2) 41 (1992), 342-352. MR 94i:42025

10. D.Termini, C.Vitanza. Weighted estimates for the Hardy-Littlewood maximal operator and Dirac deltas, Bull. London Math. Soc. 22 (1990), 367-374. MR 91f:42018

Department of Mathematics, University of Athens, Panepistimiopolis 15784, Athens, Greece

E-mail address: amelas@math.uoa.gr 